[Supporting Information]

Construction of an Artificial Biosynthetic Pathway for Zingerone Production in Escherichia coli using Benzalacetone Synthase from Piper methysticum

Kyung Taek Heo, ${ }^{1,2}$ Kyung Won Park, ${ }^{1,3}$ Juhee Won, ${ }^{1,3}$ Byeongsan Lee, ${ }^{1,3}$ Jae-Hyuk Jang, ${ }^{1,2}$ Jung-Oh Ahn, ${ }^{2,4}$ Bang Yeon Hwang, ${ }^{3}$ Young-Soo Hong,, , , *

${ }^{1}$ Anticancer Agent Research Center, Korea Research Institute of Bioscience and Biotechnology (KRIBB), 30 Yeongudanji-ro, Ochang-eup, CheongJu-si, Chungbuk 28116, Republic of Korea

${ }^{2}$ Department of Bio-Molecular Science, KRIBB School of Bioscience, University of Science and Technology(UST), Daejeon 34141, Republic of Korea

${ }^{3}$ College of Pharmacy, Chungbuk National University, Cheongju 28160, Korea

${ }^{4}$ Biotechnology Process Engineering Center, KRIBB, 30 Yeongudanji-ro, Ochang-eup, CheongJu-si, Chungbuk 28116, Republic of Korea

*Corresponding author (E-mail: hongsoo@kribb.re.kr) 
SDS-PAGE analysis. For SDS-PAGE analysis, the DZ1 and DZ4 strains were cultured under the same conditions as mentioned on Materials and Methods for the zingerone production. The harvested cell culture was re-suspended in a lysis buffer $(50 \mathrm{mM}$ Tris- $\mathrm{HCl}, 300 \mathrm{mM} \mathrm{NaCl}, 10$ $\mathrm{mM}$ imidazole, $\mathrm{pH}$ 7.4) and disrupted by sonication while chilled on ice for 10 seconds. The fully cell lysate was centrifuged at $15,000 \mathrm{rpm}$ for $10 \mathrm{~min}$. at $4{ }^{\circ} \mathrm{C}$, and the supernatant was loaded to SDS- polyacrylamide gel electrophoresis (PAGE) (12\% gel) with protein marker (10$180 \mathrm{kDa}$; BIOFACT, Korea). These gels were stained using a coomassie brilliant blue solution (Bio-Rad, USA). Additionally, the His-tagged enzymes were purified using His-Hyper Agarose resin (Lugen, Korea) in accordance with the previous reported methods. ${ }^{23}$

Prediction of zingerone structure by tandem MS analysis. The tandem was operated in electrospray ionization (ESI) mode. The typical operating parameters were as follows: spray needle voltage, $+5 \mathrm{kV}$; ion transfer capillary temperature, $275^{\circ} \mathrm{C}$; nitrogen sheath gas, 35 ; and auxiliary gas, 5 (arbitrary units). The ion trap contained helium damping gas which was introduced in accordance with the manufacturer's recommendations. Mass spectra were acquired in a $\mathrm{m} / \mathrm{z}$ range of 100-2000, with 3 microscans and a maximum ion injection time of $200 \mathrm{~ms}$. The data-dependent mass spectrometry experiments were controlled using the menu driven software provide with the Xcalibur system (version 4.0; Thermo Scientific). Raw data files were processed using Mass Frontier 7.0 software. The program modules used were Chromatogram Processor and Database Manager. Mass Frontier software was then employed to interpret MS/MS spectrum by assigning structures to the fragment ions automatically. The software Mass Frontier Spectral Interpretation (Thermo Fisher Scientific) predicts in silico fragmentation patterns to assist in the interpretation of experimental MS ${ }^{\mathrm{n}}$ data. 


\section{Legends of Supplementary Figures.}

Figure S1. Phylogenetic analysis of PmPKS against known CHS-superfamily type III PKS.

Arrow indicates PmPKS charactized in this study. The sources and GenBank accession numbers are: AtCHS from Arabidopsis thaliana (P13114), VvSTS from Vitis vinifera (ABE68894), RpBAS from Rheum palmatum (AAK82824), PmCHS from Piper methysticum (MK058495), Gh2PS from Gerbera hybrida (P48391), CHS from Medicago sativa (AAB41559), Glycine max (P19168), and Gerbera hybrida (P48390), and CTAS from Hydrangea macrophylla (BAA32733). Amino acid sequences were aligned using ClustalW method and the phylogenetic tree was constructed using the Maximum Likelihood method in MEGA7.

Figure S2. Selected mass ion chromatograms of benzalacetone compounds produced by bioconversion.

A) Peak at $7.7 \mathrm{~min}$ (Figure 3b) exhibited $\mathrm{m} / \mathrm{z} 163[\mathrm{M}+\mathrm{H}]^{+}$, corresponding to 4hydroxybenzalacetone. B) Peak at $6.5 \mathrm{~min}$ (Figure 3c) exhibited $m / z 179[\mathrm{M}+\mathrm{H}]^{+}$. C) Peak at $8.1 \mathrm{~min}$ (Figure 3d) exhibited $\mathrm{m} / \mathrm{z} 193[\mathrm{M}+\mathrm{H}]^{+}$, corresponding to vanillylidene acetone. D) Unknown peak at $8.0 \mathrm{~min}$ (Figure 3c) exhibited $\mathrm{m} / \mathrm{z} 343[\mathrm{M}+\mathrm{H}]^{+}$

Figure S3. Functional analysis of codon-optimized rzs1 gene by bioconversion experiments.

(A) HPLC profile of the authentic 4-hydroxybenzalacetone (a), authentic raspberry ketone (b) 4-hydroxybenzalacetone and raspberry ketone co-injection (c) and 4-hydroxybenzalacetone supplemented E. coli harboring pET-opRZS1 (CB2) (d) authentic vanillylidene acetone (e), authentic zingerone (f), and authentic vanillylidene acetone and zingerone co-injection (g) and vanillylidene acetone supplemented CB2 (h). Absorbance was monitored at $285 \mathrm{~nm}$. 
Figure S4. SDS-PAGE analysis for the expression of zingerone biosynthetic enzymes in E. coli strains (DZ1 and DZ4)

M, Middle molecular weight protein marker; Lane 1, His-tagged 4CL (61.6 kDa); Lane 2, Histagged RZS1 (40.6 kDa); Lane 3, His-tagged PmPKS (45.6 kDa); Lane 4 and 5 were the supernatants of DZ1 (harboring pET22-opT5M and pET-4RPm) and DZ4 (pET22-opT5M and pACYCD-4RPm) strains, respectively; Lane 6 and 7 were the total cell lysate of DZ1 and DZ4 strains, respectively.

Figure S5. Scheme representation of construction of the expression vectors, pET-4Pm, pET-4RPm, and pACYCD-4RPm.

Figure S6. HPLC profile under modified elution condition for quantification of zingerone.

A) HPLC profile of the culture broth of DZ6 strain, B) HPLC profile of the authentic zingerone and vanillylidene acetone. The zingerone and vanillylidene acetone peaks were obtained at $19.9 \mathrm{~min}$, and $22.3 \mathrm{~min}$ in modified condition, respectively.

Figure S7. Comparison tandem mass spectra of the standard zingerone (A) and produced zingerone peak from the culture extract $(B)$.

MS (a), $\mathrm{MS}^{2}(\mathrm{~b})$, and $\mathrm{MS}^{3}$ (c) spectra of standard zingerone and produced zingerone peak. Each upper panels (a) are MS spectra of each compound. Middle panels (b) are $\mathrm{MS}^{2}$ spectra of $m / z$ 195.22 (A) and 195.25 (B), respectively. Lower panels (c) are $\mathrm{MS}^{3}$ spectra of $m / z 136.93$ (A) and $136.92(\mathrm{~B})$, respectively. 
Figure S1. Phylogenetic analysis of PmPKS against known CHS-superfamily type III PKS.

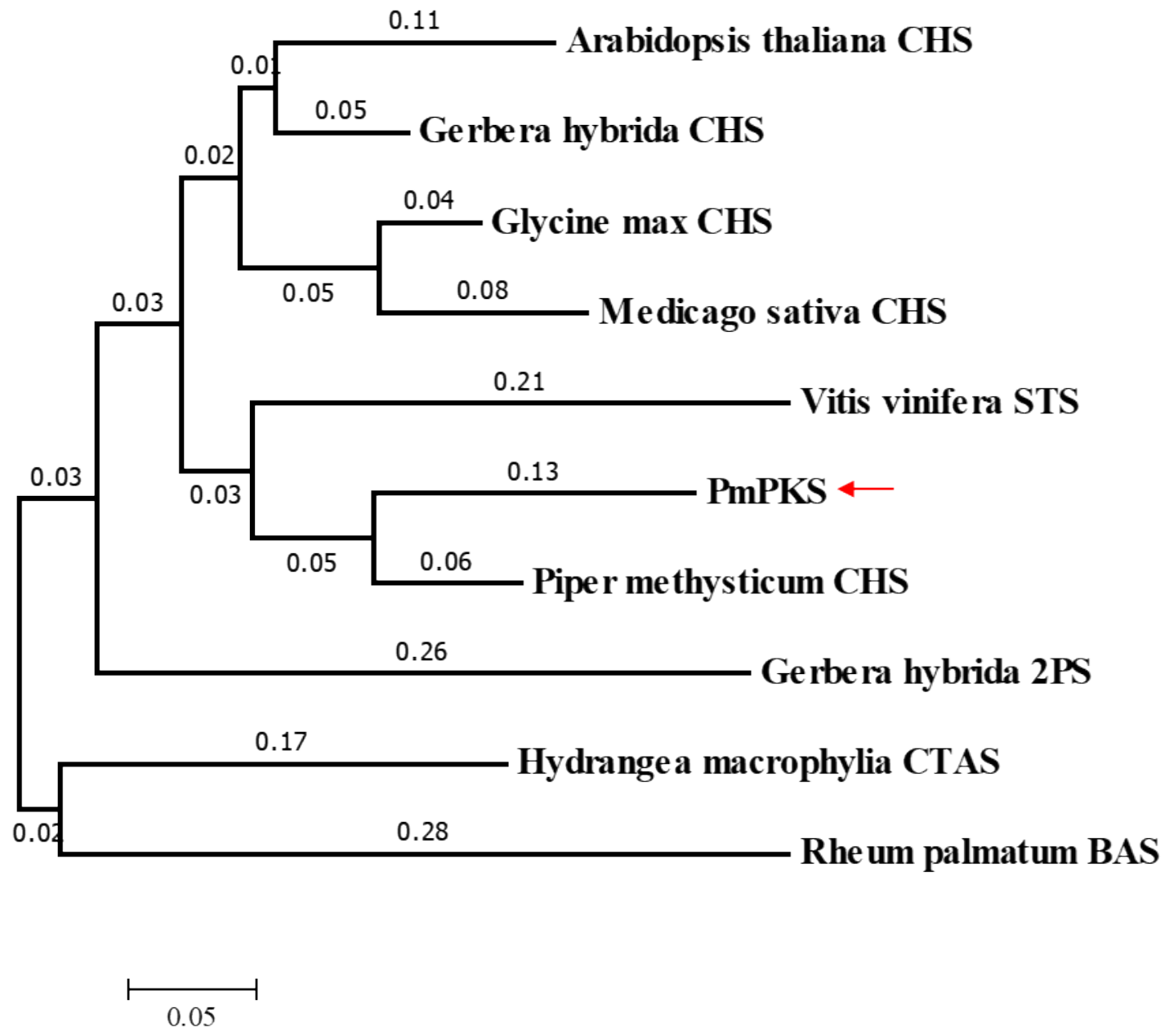


Figure S2. Selected mass ion chromatograms of benzalacetone compounds produced by bioconversion.
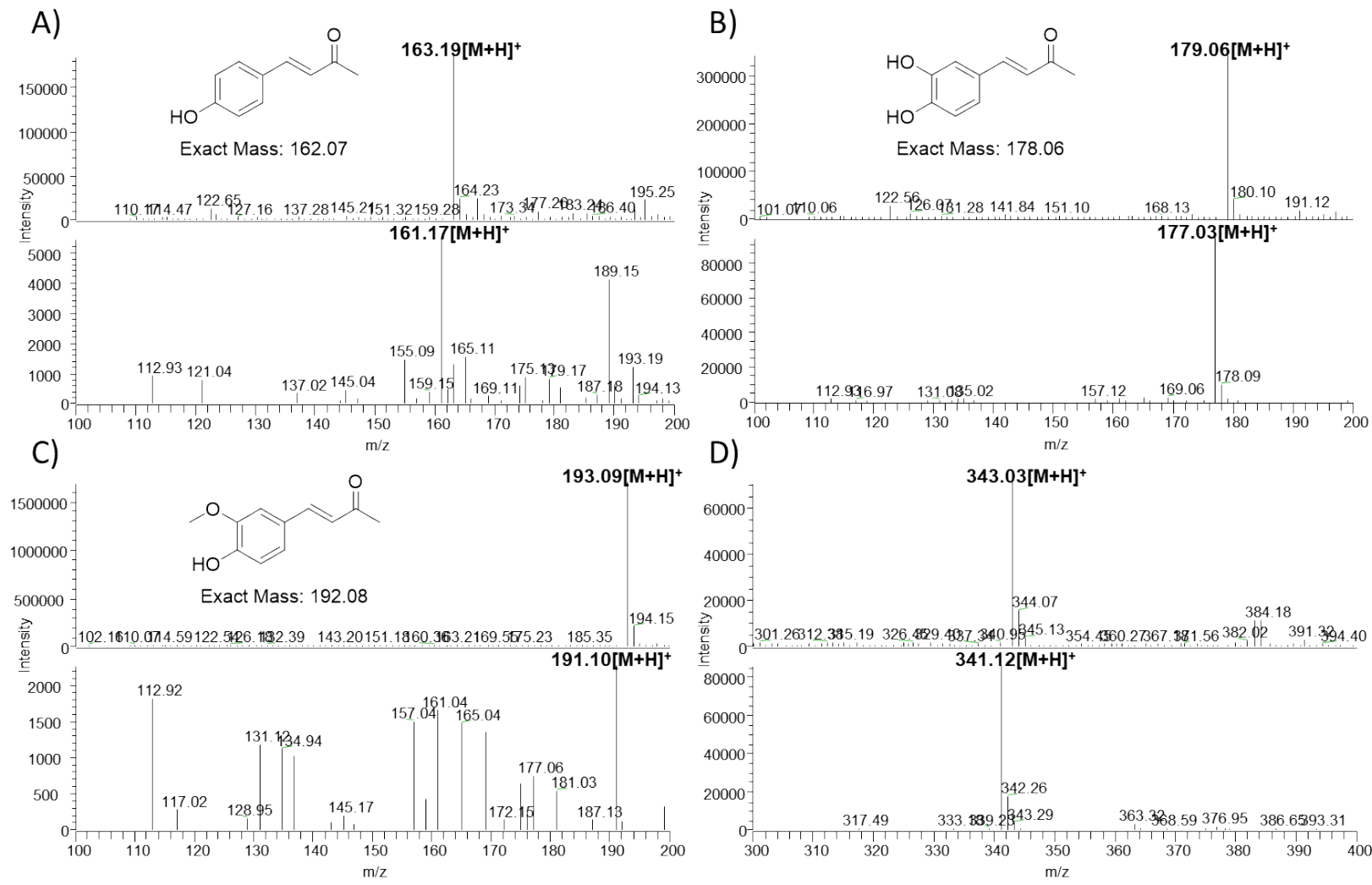
Figure S3. Functional analysis of codon-optimized rzs1 gene by bioconversion experiments.

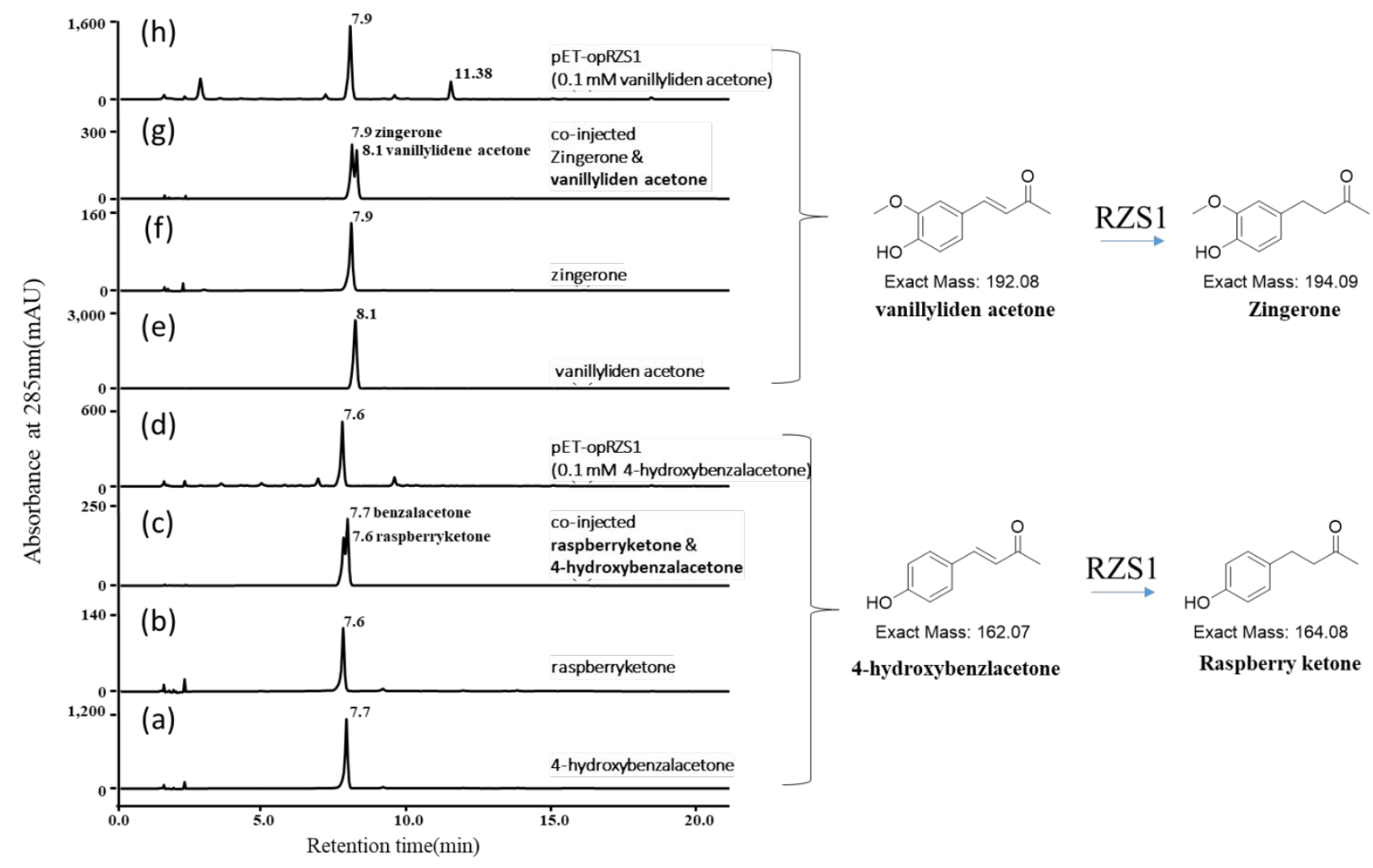


Figure S4. SDS-PAGE analysis for the expression of zingerone biosynthetic enzymes in E. coli strains (DZ1 and DZ4)

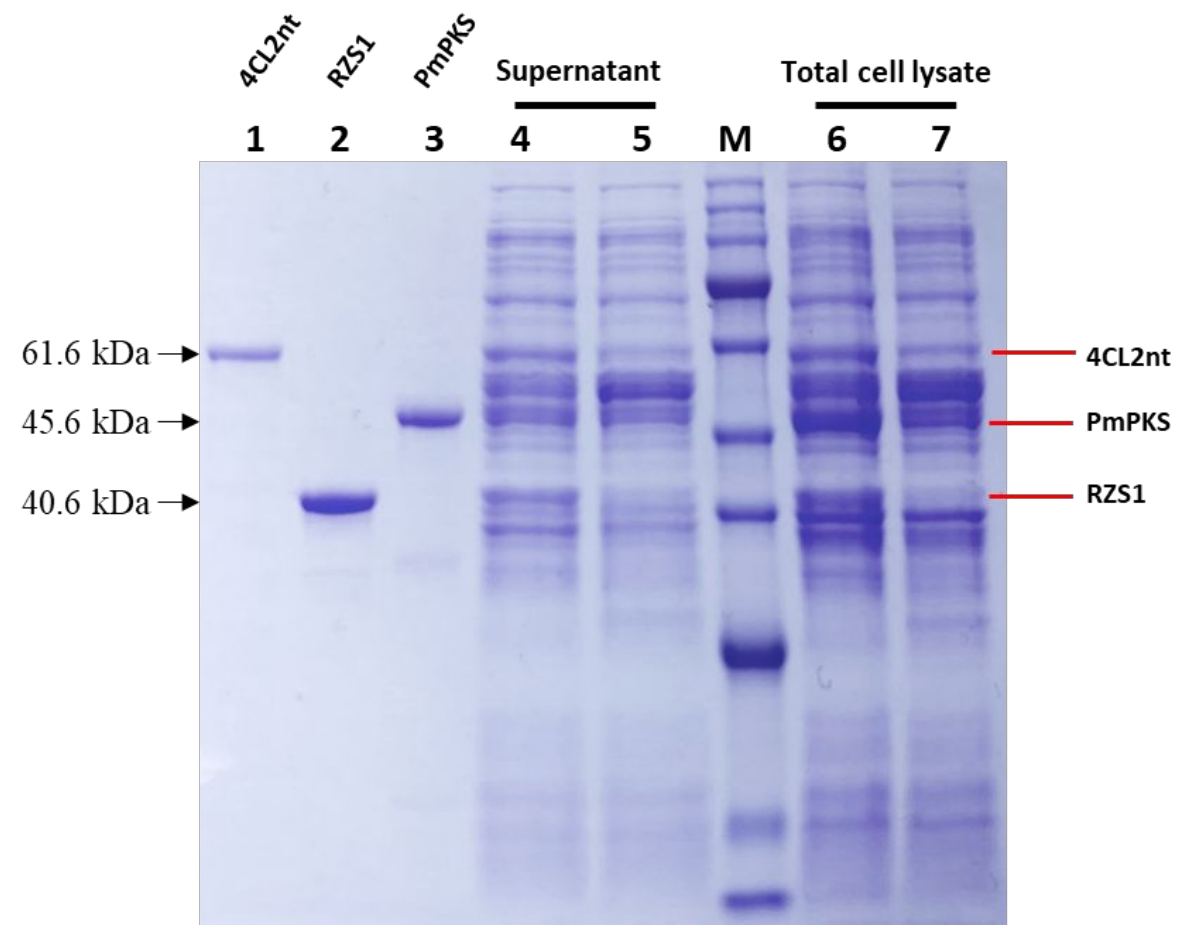


Figure S5. Scheme representation of construction of the expression vectors, pET-4Pm, pET-4RPm, and pACYCD-4RPm.
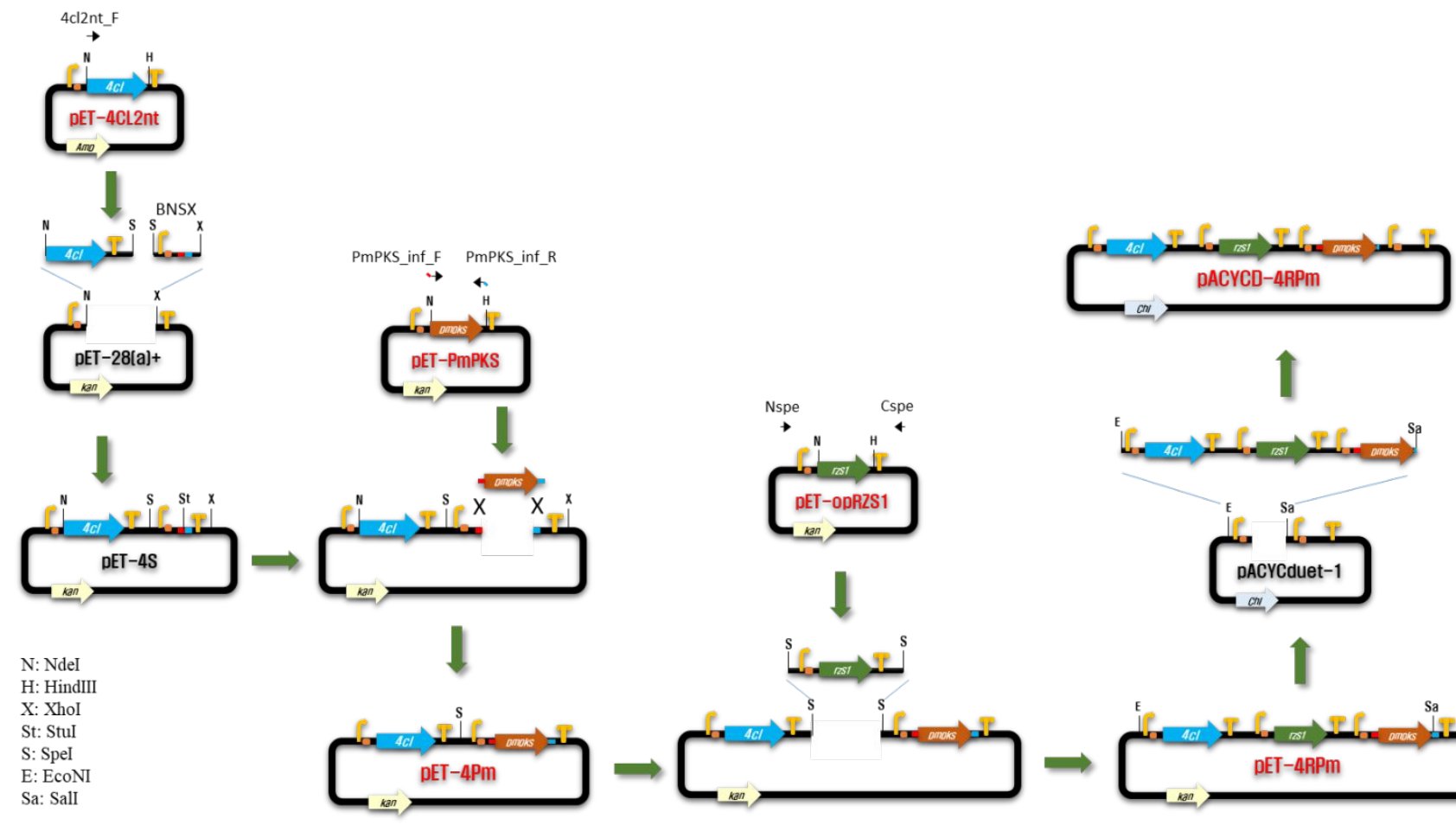
Figure S6. HPLC profile under modified elution condition for quantification of zingerone.

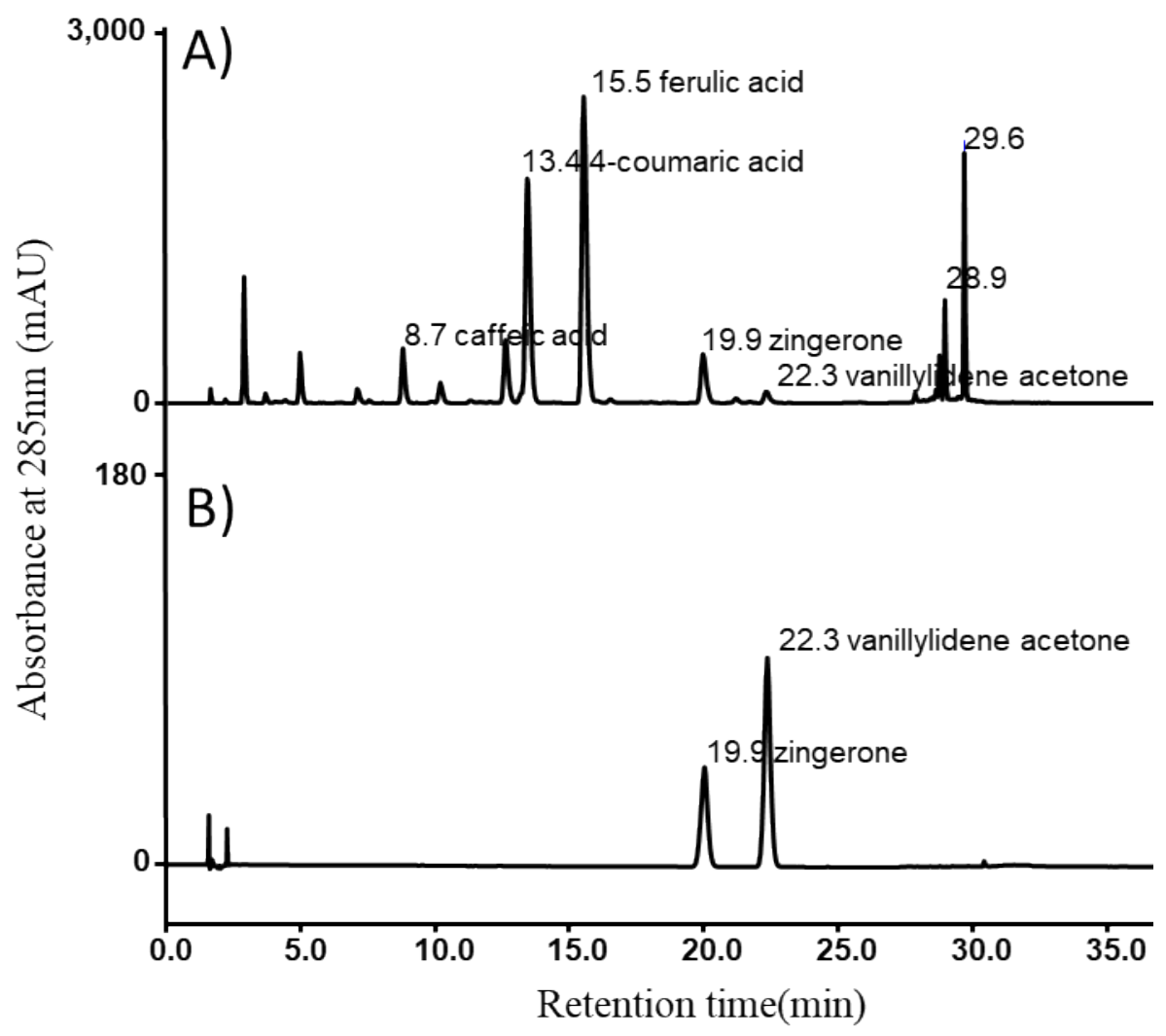


Figure S7. Comparison tandem mass spectra of the standard zingerone (A) and produced zingerone peak from the culture extract $(B)$.

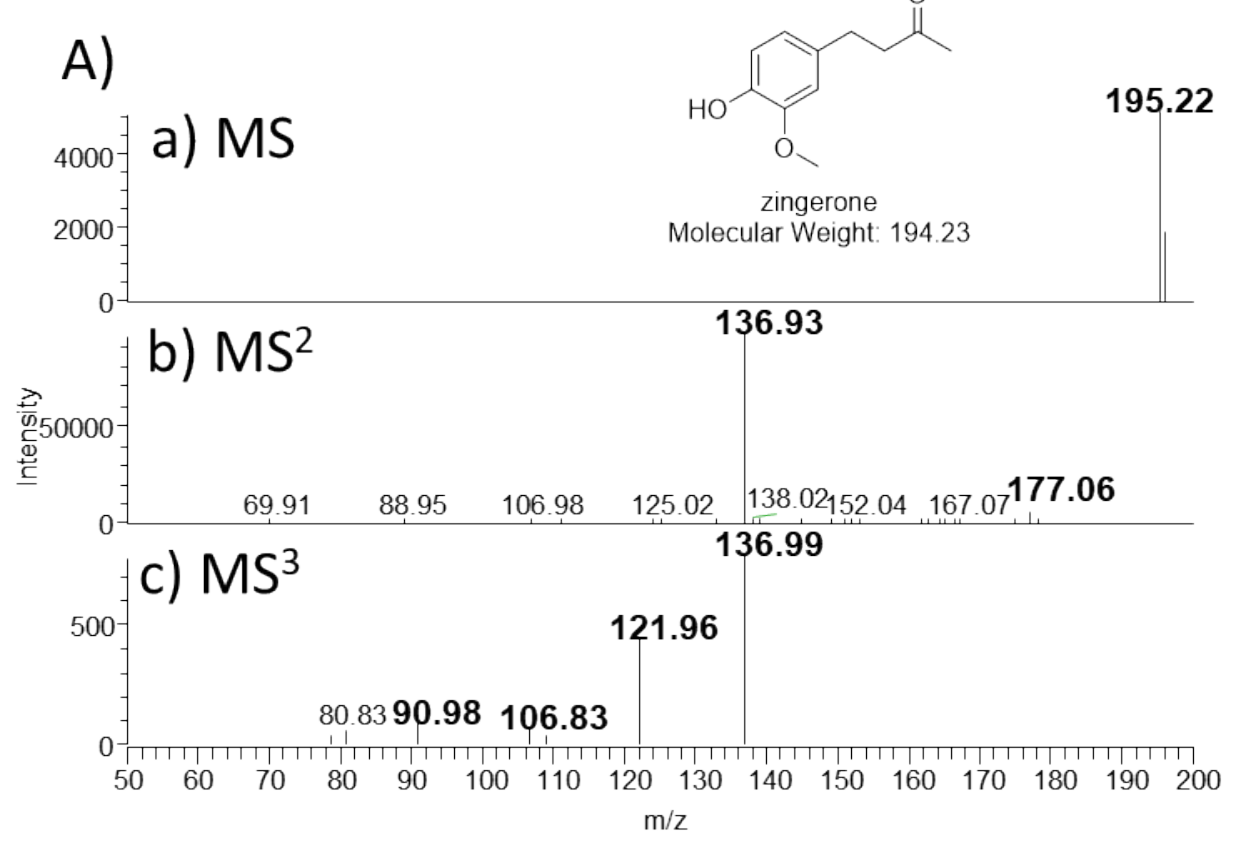

B)

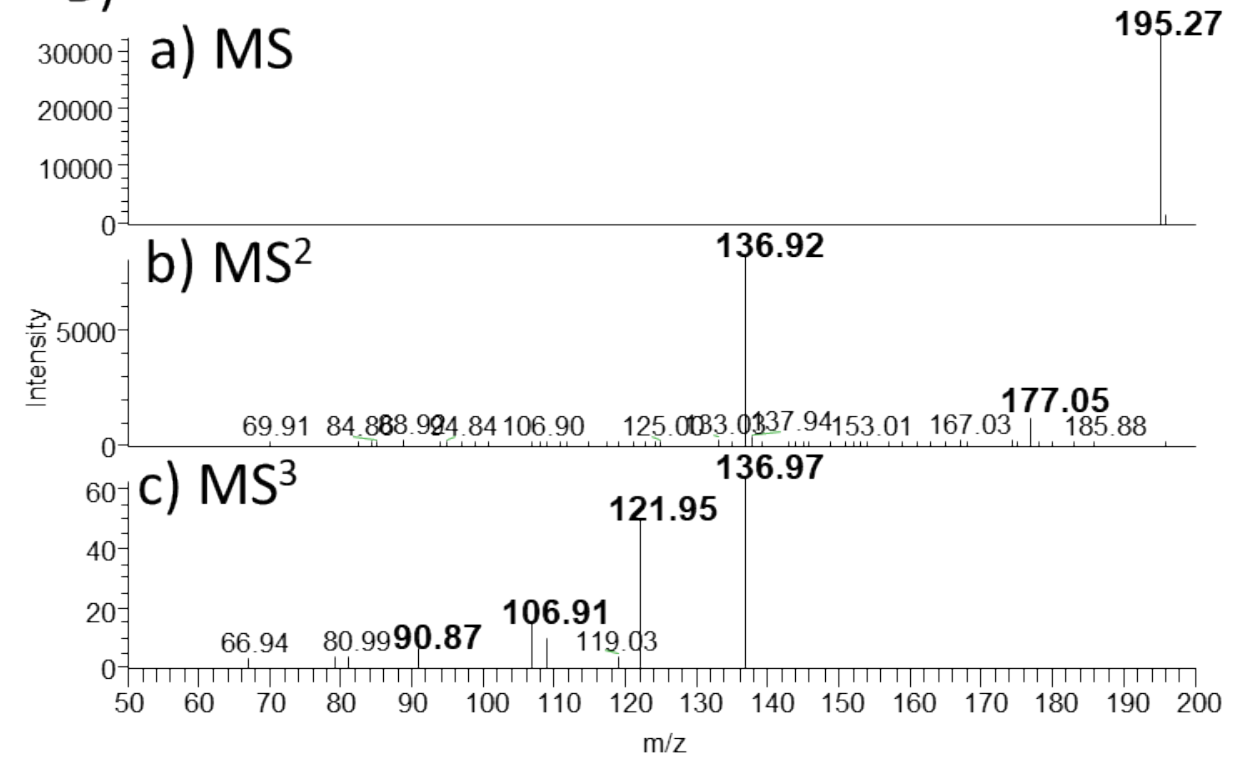


> codon-optimized pmpks gene

ATGAGCAAAACCGTTGAAGATCGTGCAGCACAGCGTGCAAAAGGTCCGGCAACCGTTCTGGCAATT GGTACCGCAACCCCGGCAAATGTTGTTTATCAGACCGATTATCCGGATTATTATTTTCGTGTTACCAAA AGCGAACACATGACCAAACTGAAAAATAAATTTCAGCGTATGTGTGATCGTAGCACCATTAAAAAAC GTTATATGGTTCTGACCGAAGAACTGCTGGAAAAAAATCTGAGCCTGTGTACCTATATGGAACCGAG CCTGGATGCACGTCAGGATATTCTGGTTCCGGAAGTTCCGAAACTGGGTAAAGAAGCAGCAGATGAA GCAATTGCAGAATGGGGTCGTCCGAAAAGCGAAATTACCCATCTGATTTTTTGTACCACCTGTGGTGT TGATATGCCGGGTGCAGATTATCAGCTGACCAAACTGCTGGGTCTGCGTAGCAGCGTTCGTCGTACC ATGCTGTATCAGCAGGGTTGTTTTGGTGGTGGTACCGTTCTGCGTCTGGCAAAAGATCTGGCAGAAAA TAATGCAGGTGCACGTGTTCTGGTTGTTTGTAGCGAAATTACCACCGCAGTTAATTTTCGTGGTCCGA GCGATACCCATCTGGATCTGCTGGTTGGTCTGGCACTGTTTGGTGATGGTGCAGCAGCAGTTATTGTT GGTGCAGATCCTGATCCGACCCTGGAACGTCCGCTGTTTCAGATTGTTAGCGGTGCACAGACCATTCT GCCGGATAGCGAAGGTGCAATTAATGGTCATCTGCGTGAAGTTGGTCTGACCATTCGTCTGCTGAAA GATGTTCCGGGTCTGGTTAGCATGAATATTGAAAAATGTCTGATGGAAGCATTTGCACCGATGGGTAT TCATGATTGGAATAGCATTTTTTGGATTGCACATCCGGGTGGTCCGACCATTCTGGATCAGGTTGAAG CAAAACTGGGTCTGAAAGAAGAAAAACTGAAAAGCACCCGTGCAGTTCTGCGTGAATATGGTAATAT GAGCAGCGCATGTGTTCTGTTTATTCTGGATGAAGTTCGTAAACGTAGCATGGAAGAAGGTAAAACC ACCACCGGTGAAGGTTTTGATTGGGGTGTTCTGTTTGGTTTTGGTCCGGGTTTTACCGTTGAAACCGTT GTTCTGCATAGCATGCCGATTCCGAAAGCAGATGAAGGTCGTTAA

> codon-optimized rzs1 gene

ATGGCCTCAGGTGGTGAGATGCAAGTTTCAAACAAGCAAGTCATCTTCCGTGATTATGTTACCGGTTTTCCG AAAGAAAGCGATATGGAACTGACCACCCGTAGCATTACCCTGAAACTGCCGCAGGGTAGCACCGGTCTGC TGCTGAAAAATCTGTATCTGAGCTGTGATCCGTATATGCGTGCACGTATGACCAATCATCATCGTCTGAGCT ATGTTGATAGCTTTAAACCGGGTAGCCCGATTATTGGTTATGGTGTTGCACGTGTTCTGGAAAGCGGTAATC CGAAATTTAATCCGGGTGATCTGGTTTGGGGTTTTACCGGTTGGGAAGAATATAGCGTTATTACCGCAACC GAAAGCCTGTTTAAAATTCATAATACCGATGTTCCGCTGAGCTATTATACCGGTCTGCTGGGTATGCCGGGT ATGACCGCCTACGCAGGTTTTTATGAAATTTGTAGCCCGAAAAAAGGTGAAACCGTTTATGTTAGCGCAGC AAGCGGTGCAGTTGGTCAGCTGGTTGGTCAGTTTGCAAAACTGACCGGTTGTTATGTTGTTGGTAGCGCAG GTAGCAAAGAAAAAGTTGATCTGCTGAAGAATAAGTTTGGTTTCGATGAAGCATTCAATTATAAAGAAGAA GCAGATCTGGATGCAGCACTGCGTCGTTATTTTCCGGATGGTATTGACATCTACTTCGAGAATGTTGGTGGC AAGATGCTGGATGCAGTTCTGCCGAATATGCGTCCGAAAGGTCGTATTGCAGTTTGTGGTATGATTAGCCA GTATAATCTGGAACAGCCGGAAGGTGTTCGTAATCTGATGGCACTGATTGTTAAACAGGTTCGTATGGAAG GTTTTATGGTTTTTAGCTATTATCATCTGTATGGTAAATTTCTGGAAACCGTTCTGCCGTATATTAAACAGGG TAAAATTACCTATGTTGAAGATGTTGTTGATGGTCTGGATAATGCACCGGCAGCACTGATTGGTCTGTATAG CGGTCGTAATGTTGGTAAACAGGTTGTTGTTGTTAGCCGTGAATAA 